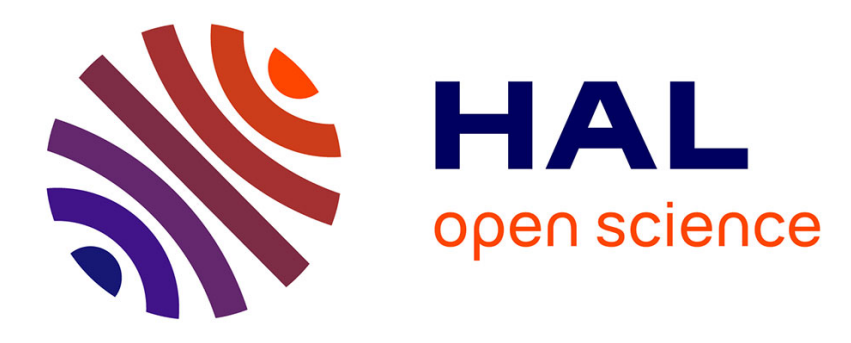

\title{
Direct numerical simulation of axisymmetric turbulence
} Bo Qu, Wouter J.T. Bos, Aurore Naso

\section{- To cite this version:}

Bo Qu, Wouter J.T. Bos, Aurore Naso. Direct numerical simulation of axisymmetric turbulence. Physical Review Fluids, 2017, 2 (9), 10.1103/PhysRevFluids.2.094608 . hal-01559042v2

\section{HAL Id: hal-01559042 \\ https://hal.science/hal-01559042v2}

Submitted on 9 Apr 2019

HAL is a multi-disciplinary open access archive for the deposit and dissemination of scientific research documents, whether they are published or not. The documents may come from teaching and research institutions in France or abroad, or from public or private research centers.
L'archive ouverte pluridisciplinaire HAL, est destinée au dépôt et à la diffusion de documents scientifiques de niveau recherche, publiés ou non, émanant des établissements d'enseignement et de recherche français ou étrangers, des laboratoires publics ou privés. 


\title{
Direct numerical simulation of axisymmetric turbulence
}

\author{
Bo Qu, Wouter J. T. Bos, and Aurore Naso \\ Laboratoire de Mécanique des Fluides et d'Acoustique, CNRS, École Centrale de Lyon, Université de Lyon, \\ INSA de Lyon, 36 avenue Guy de Collongue, 69134 Écully Cedex, France
}

(Received 20 March 2017; published 25 September 2017)

\begin{abstract}
The dynamics of decaying, strictly axisymmetric, incompressible turbulence is investigated using direct numerical simulations. It is found that the angular momentum is a robust invariant of the system. It is further shown that long-lived coherent structures are generated by the flow. These structures can be associated with stationary solutions of the Euler equations. The structures obey relations in agreement with predictions from selective decay principles, compatible with the decay laws of the system. Two different types of decay scenarios are highlighted. The first case results in a quasi-two-dimensional flow with a dynamical behavior in the poloidal plane similar to freely decaying two-dimensional turbulence. In a second regime, the long-time dynamics is dominated by a single three-dimensional mode.
\end{abstract}

DOI: 10.1103/PhysRevFluids.2.094608

\section{INTRODUCTION}

The successful development of a statistical mechanics approach to describe turbulent flows started with the work of Burgers [1] and Onsager [2,3] on the description of two-dimensional (2D) turbulence using point vortices. Subsequent theoretical developments of Joyce and Montgomery [4-6] and Lundgren and Pointin [7] formalized Onsager's ideas (see also the review by Kraichnan and Montgomery [8]). It was shown in simulations of decaying two-dimensional Navier-Stokes turbulence that the theoretical predictions of the point vortex model were in some cases surprisingly accurately verified [9]. Indeed, the presence of viscous dissipation did not seem to invalidate the statistical mechanics approach derived for a discrete inviscid system.

To go beyond the point-vortex case, in order to apply statistical mechanics to the continuum case, Miller [10] and Robert and Sommeria [11] simultaneously introduced a coarse-grained description of the vorticity field. Introducing a mixing entropy and maximizing it under constraints of conserved energy and other invariants, they managed to extend the statistical mechanics approach to the case of the two-dimensional Euler equations. The resulting Miller-Robert-Sommeria (MRS) theory predicts that the final states obtained by integrating the decaying 2D Euler equations should be characterized, in the limit of strong mixing [12], or accounting for the conservation of the lowest order invariants only [13], by a linear relationship between the coarse-grained streamfunction $\psi$ and vorticity $\omega$. In the general case, any monotonic relation between these quantities can be obtained. These predictions from equilibrium statistical mechanics are reasonably well satisfied by a number of direct numerical simulations of the 2D Euler equations, even though some of these simulations raised some doubts about the stationarity of the final states, their nondependence on the initial conditions, and the validity of the underlying ergodicity hypothesis [14-16].

Subsequently, using the same tools, explanations were given for the behavior of the shallow water system [17-20] and the Great Red Spot on Jupiter [21,22]. In these situations, the inertial terms dominate, and the dynamics can be considered as governed by the incompressible Euler equations. Similar approaches were used to describe the large-scale oceanic currents on Earth [23-25], although it should be stressed here that the time average of real oceanic flows at the basin scale, away from boundary currents, is dominated by the wind forcing.

Obviously one would like to apply these ideas to the more general case of three-dimensional (3D) turbulence, but the dissipative anomaly, i.e., a nonvanishing energy dissipation in the limit of vanishing viscosity (see, for instance, Refs. [26,27] for experimental and numerical evidence) distinguishes drastically the physics of three-dimensional homogeneous and isotropic turbulence 
from the two-dimensional case. However, specific cases of three-dimensional flows, where the large-scale structure is closer to the two-dimensional case, might be amenable to treatment by statistical mechanics.

This possibility motivated a number of studies on axisymmetric turbulence, i.e., axisymmetric flows in cylindrical geometry. Indeed, the axisymmetric Euler (or Navier-Stokes) equations describe the coupled dynamics of three velocity components varying along two spatial directions. This situation is therefore intermediate between two- and three-dimensional flows. Similarly to what occurs in 2D turbulence, the nonlinear dynamics of axisymmetric turbulence is constrained by the existence of an infinite number of Casimir invariants, quantities conserved in the limit of vanishing viscosity and forcing. Different theories based on statistical mechanics at equilibrium were developed to characterize the general stationary solutions of the axisymmetric Euler equations. However, a full theory cannot be built without modeling the statistics of this system at small scale. Mohseni [28] and Lim [29] first considered nonswirling flows and obtained results very similar to those obtained in the $2 \mathrm{D}$ case. Another theoretical framework was built by Leprovost and collaborators [30] and further developed by Naso et al. [31,32], assuming that the poloidal degrees of freedom do not fluctuate. More recently, Thalabard et al. [33] defined another theoretical framework, in a Taylor-Couette geometry, in which a cutoff is imposed on the poloidal degrees of freedom as an intermediate regulation constraint (incidently, such a cutoff had been introduced in Ref. [30] as a physical parameter to go beyond the hypothesis of nonfluctuating poloidal flow). We stress here the fact that vorticity is not only mixed but also stretched by the axisymmetric Navier-Stokes equations. This is a crucial difference with 2D turbulence: The formation of finite-time singularities in flows described by the axisymmetric Euler equations is in particular very likely to occur [34,35]. This might be an issue for describing axisymmetric turbulence with theories based on the conservation of dynamical invariants, but such approaches are nevertheless interesting at a phenomenological level.

In practice, it is very difficult to generate a real turbulent flow which will remain invariant along the azimuthal direction. An exception might be turbulence in the presence of a strong azimuthal magnetic field, but this kind of flows, encountered in magnetically confined nuclear fusion devices such as tokamaks, have other experimental difficulties to cope with. Only on average experimental flows are sometimes axisymmetric. This is the case of the von Kármán flow, a cylindrical flow between rotating plates, or impellers [36]. The (axisymmetric) time average of this flow surprisingly displays some features predicted by the abovementioned theoretical developments. The experiments by Ravelet et al. [37] showed that large-scale structures similar to those predicted by the theories form in this mean flow. Later on, Monchaux et al. [38] observed that at large values of the Reynolds number, the stationary state of the time-averaged flow tends to select the shape of a Beltrami-flow pattern [39] (a dominant alignment of velocity and vorticity), which is a specific solution of the variational problem. More recently, Thalabard et al. [40] showed that the large-scale structures observed in the mean von Kármán flow could be interpreted by using an equilibrium theory relying on the axisymmetric Euler equations together with an adequate modeling of the fluctuations.

However, the theory was developed for purely axisymmetric flows, which can only be precisely reproduced in numerical simulations. To the best of our knowledge, such simulations have never been carried out and the present investigation aims at giving the first results using direct numerical simulations (DNS) of the axisymmetric Navier-Stokes equations. It can be noticed here that DNS of the axisymmetric Euler equations have previously been performed (see, e.g., Refs. [34,35]), with the aim of seeking the formation of finite-time singularities. One can also mention the direct numerical simulation [41] of the Navier-Stokes equations with helical symmetry, which includes in principle the axial symmetry in the limit of vanishing helix pitch, but such a limiting case was not treated in this work.

In the following section, we will recall the equations describing the system and the invariants of the inviscid system, and we will summarize the main theoretical predictions that we will assess. Then, in Sec. III, we will describe the numerical method and the initial conditions of our simulations. In Sec. IV, we present the results, which are briefly discussed in Sec. V. In Sec. VI, the conclusions and perspectives are summarized. 


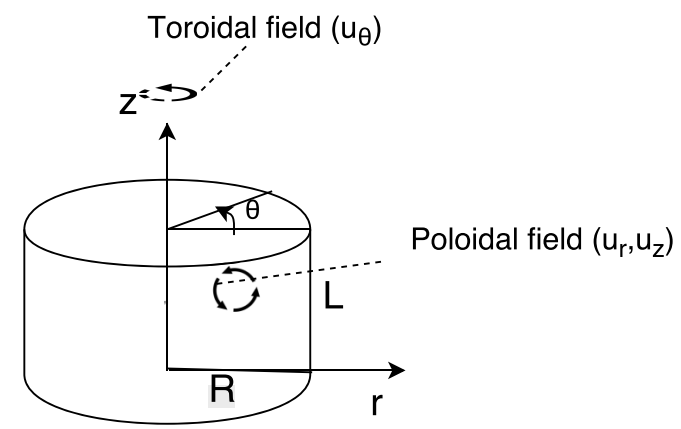

FIG. 1. System of coordinates and computational domain.

\section{AXISYMMETRIC TURBULENCE AND PREDICTIONS FROM STATISTICAL MECHANICS}

\section{A. Equations and invariants}

In this investigation, we consider the incompressible axisymmetric Navier-Stokes equations in cylindrical coordinates $(r, \theta, z)$, for the three velocity components $\left(u_{r}, u_{\theta}, u_{z}\right)$. The domain and the coordinates are illustrated in Fig. 1.

The difference with the full Navier-Stokes equations is that our system is axisymmetric, that is, in the equations all the azimuthal derivatives $\partial / \partial \theta$ are zero. For instance, the incompressibility condition becomes

$$
\frac{1}{r} \frac{\partial}{\partial r}\left(r u_{r}\right)+\frac{\partial u_{z}}{\partial z}=0
$$

The goal of the present work is to test the predictions obtained using tools borrowed from statistical mechanics. Such predictions are obtained from considerations on the invariants of the Euler equations, i.e., quantities which are conserved by the system in the absence of viscous dissipation. We therefore consider in this section the axisymmetric Euler equations.

It was shown in Ref. [30] that using a change of variables, the Euler equations take the simple form of two coupled equations for scalar quantities. The first of these scalar quantities is the potential vorticity,

$$
\xi=\frac{1}{r}\left(\partial_{z} u_{r}-\partial_{r} u_{z}\right)=\frac{\omega_{\theta}}{r}
$$

where the vorticity is defined by $\boldsymbol{\omega}=\nabla \times \boldsymbol{u}$. The second quantity is the angular momentum $\sigma=r u_{\theta}$. The potential vorticity $\xi$ can be computed from a stream function using the generalized Laplacian, $\Delta_{*}$,

$$
\Delta_{*} \psi \equiv \frac{1}{2 y} \partial_{z}^{2} \psi+\partial_{y}^{2} \psi=-\xi
$$

where the variable $y$ is defined as $y=r^{2} / 2$.

The advantage of these definitions is that the Euler equations then reduce to

$$
\begin{aligned}
\partial_{t} \sigma+\{\psi, \sigma\} & =0, \\
\partial_{t} \xi+\{\psi, \xi\} & =\partial_{z}\left(\frac{\sigma^{2}}{4 y^{2}}\right),
\end{aligned}
$$

where the Poisson brackets are defined classically, as

$$
\{\psi, \phi\}=\partial_{y} \psi \partial_{z} \phi-\partial_{z} \psi \partial_{y} \phi
$$


We have hereby defined a two-dimensional system, described by $\sigma, \xi$, as a function of the variables $y, z$.

The invariants of this system are the energy,

$$
E=\frac{1}{2}\langle\xi \psi\rangle+\frac{1}{2}\left\langle\frac{\sigma^{2}}{r^{2}}\right\rangle,
$$

all moments of the angular momentum,

$$
I_{m}=\left\langle\sigma^{m}\right\rangle \quad(m \geqslant 1)
$$

and generalized helicities, defined by

$$
H_{n}=\left\langle\xi \sigma^{n}\right\rangle \quad(n \geqslant 0),
$$

where \langle\rangle$=1 /\left(\pi R^{2} L\right) \int_{0}^{R} r d r \int_{0}^{2 \pi} d \theta \int_{0}^{L} d z$ is the spatial average over a cylindrical domain of radius $R$ and height $L$. We will hereafter focus on the first invariants, $E, I_{1}=\langle\sigma\rangle$ (angular momentum), $I_{2}=\left\langle\sigma^{2}\right\rangle, H_{0}=\langle\xi\rangle$ (circulation), and $H_{1}=\langle\sigma \xi\rangle=\left\langle u_{\theta} \omega_{\theta}\right\rangle$ (helicity) [42]. Note that the energy contains two separate parts, the poloidal and the toroidal energies, respectively,

$$
\begin{aligned}
& E_{P}=\frac{1}{2}\langle\xi \psi\rangle, \\
& E_{T}=\frac{1}{2}\left\langle\frac{\sigma^{2}}{r^{2}}\right\rangle .
\end{aligned}
$$

We note here a degenerate case, important for the interpretation of the results obtained in this work. If in the above system $\sigma$ tends to zero (nonswirling flow), or more generally, the right-hand side (RHS) of Eq. (5), $\partial_{z}\left(\sigma^{2} /\left(4 y^{2}\right)\right)=0$, then the dynamics of $\sigma$ and $\xi$ are decoupled, and Eq. (5) becomes a pure mixing equation for $\xi$, identical to the 2D Euler equations. In this case, new invariants appear [28,29], i.e., Casimirs of the potential vorticity

$$
\Gamma_{n}=\left\langle\xi^{n}\right\rangle \quad(n \geqslant 0)
$$

are conserved. In this two-dimensional limit, the invariants that will play the most important role are the poloidal energy $E_{P}$ and the potential enstrophy

$$
Z=\left\langle\xi^{2}\right\rangle
$$

\section{B. Theoretical predictions}

Two families of predictions are possible in the light of the foregoing considerations. First a dynamics similar to two-dimensional turbulence [governed by Eq. (5) with zero RHS], where the azimuthal component of the velocity is either zero (nonswirling case) or invariant with respect to the $z$ direction, or a system where $\sigma$ and $\xi$ are coupled and the most probable state should obey the two equations (4) and (5) simultaneously.

\section{Two-dimensional turbulence in the poloidal plane (including nonswirling flows: $\sigma=0$ )}

In the case of small, or axially invariant $\sigma$, the dynamics described by Eqs. (4) and (5) reduces to

$$
\partial_{t} \xi+\{\psi, \xi\}=0
$$

coupled with Eq. (3). The invariants of this two-dimensional system, very similar to the 2D Euler equations, are the poloidal energy and the Casimirs of the potential vorticity, $\left\langle\xi^{n}\right\rangle[28,29]$. Its stationary solutions trivially obey the relation

$$
\xi=f(\psi)
$$


where $f(\psi)$ is an arbitrary function whose shape is to be determined. It will be shown hereafter that this quasi-two-dimensional poloidal dynamics can indeed be observed in decaying axisymmetric turbulence.

\section{Fully axisymmetric turbulence (swirling flows: $\sigma \neq 0$ )}

In the nondegenerate case of fully axisymmetric turbulence, steady solutions of the axisymmetric Euler equations, obtained by setting $\partial_{t} \sigma$ and $\partial_{t} \xi$ to zero in Eqs. (4) and (5), were shown [30] to obey the following relations among $\sigma, \xi$, and $\psi$ :

$$
\begin{gathered}
\sigma=F(\psi), \\
\xi-\frac{F(\psi) F^{\prime}(\psi)}{r^{2}}=G(\psi),
\end{gathered}
$$

where $F$ and $G$ are arbitrary functions of $\psi$.

If equilibrium states, metastable for times shorter than the viscous diffusion time of the flow, exist, they are thus expected to satisfy these expressions. The shape of these expressions cannot be concluded directly from these equations without further assumptions. In the spirit of investigations of two-dimensional turbulence [12,13], it can be shown that the expressions of $F$ and $G$ depend on a number of invariants whose conservation is supposed to play an important role in the dynamics [31,32]. In some cases, the solutions of the resulting variational principle are the same as those of a specific selective decay principle [13,31]. In particular, it was shown [31] that in the axisymmetric case the maximization of entropy at fixed helicity, angular momentum, and microscopic energy is equivalent to the minimization of macroscopic energy at fixed helicity and angular momentum. The solutions of these variational problems are such that $F$ is a linear function and $G$ is a constant. The resulting flows are in a Beltrami state, i.e., the vorticity and the velocity are aligned everywhere.

The states described by Eqs. (15) (nonswirling flow) or (16) and (17) (swirling flow) are expected to be metastable, for specific choices of the functions $f$, or $F$ and $G$, as a consequence of the Arnold energy-Casimir method [43]. One of the aims of the present work is to check whether, in the case of finite viscosity, meta-stable states are observed in which the flow obeys the expressions (15), or (16) and (17).

\section{NUMERICAL METHOD AND INITIAL CONDITIONS}

\section{A. Eigenfunctions of the curl}

The numerical method we use to study strictly axisymmetric turbulence is fully spectral. We modified and further developed an existing code, solving the Navier-Stokes equations in a circular geometry [44-46], based on an expansion of the velocity field using Chandrasekhar-Kendall helical eigenfunctions of the curl [47]. These functions form a complete and orthogonal basis in cylindrical geometry. This type of method has been previously applied to the numerical study of 2D turbulence in a circular geometry [44-46] and of 3D magnetohydrodynamics in cylindrical [48-51] and spherical [52] geometries. In the axisymmetric case, the same modal decomposition was used in Ref. [31] (their subsection V.C.1) to calculate the solutions of a simplified thermodynamics approach of the axisymmetric Euler equations, and in Ref. [53] to reconstruct experimentally measured velocity and magnetic fields in the von Kármán experiment. We use radial nonpenetration and axially periodic boundary conditions, since those are the most natural conditions using our method, and since they correspond to a physically interesting case, where the axial motion is not constrained by solid boundaries.

Velocity fields are projected on a basis consisting of Chandrasekhar-Kendall eigenfunctions of the curl $\mathbf{A}_{n q}$, defined such that

$$
\nabla \times \boldsymbol{A}_{n q}=\lambda_{n q} \boldsymbol{A}_{n q} .
$$


The solutions of this equation satisfying the suitable boundary conditions are a combination of Fourier modes in the axial direction and Bessel functions in the radial one:

$$
\boldsymbol{A}_{n q}=I_{n q}^{-\frac{1}{2}}\left(\begin{array}{c}
k_{n} \gamma_{q} J_{1}\left(\gamma_{q} r\right) i e^{-i k_{n} z} \\
\lambda_{n q} \gamma_{q} J_{1}\left(\gamma_{q} r\right) e^{-i k_{n} z} \\
\gamma_{q}^{2} J_{0}\left(\gamma_{q} r\right) e^{-i k_{n} z}
\end{array}\right)
$$

where $I_{n q}^{-\frac{1}{2}}$ is a normalization coefficient ensuring that $\left\langle\boldsymbol{A}_{n q} \boldsymbol{A}_{n q}^{*}\right\rangle=1, k_{n}=2 \pi n / L \quad(n=$ $0, \pm 1, \pm 2, \ldots)$, and $\gamma_{q}$ is the $q$ th zero of the function $f(x)=J_{1}(R x)$. Finally, the following relation is satisfied:

$$
\lambda_{n q}^{2}=\gamma_{q}^{2}+k_{n}^{2} .
$$

Both signs of $\lambda_{n q}$ must be accounted for. The functions $\mathbf{A}_{n q}$ form an orthonormal basis of solenoidal functions, allowing a precise, nondiffusive representation of the dynamics of the velocity field, represented by the coefficients $\xi_{n q}(t)$,

$$
\mathbf{u}(r, z, t)=\sum_{n q} \xi_{n q}(t) \mathbf{A}_{n q}(r, z)
$$

In the basis of Chandrasekhar-Kendall modes, the Navier-Stokes equations can be written as

$$
\frac{\partial}{\partial t} \xi_{n q}^{v}=\sum_{n^{\prime} q^{\prime}} \sum_{n^{\prime \prime} q^{\prime \prime}} \lambda_{n^{\prime \prime} q^{\prime \prime}} \xi_{n^{\prime} q^{\prime}}^{v} \xi_{n^{\prime \prime} q^{\prime \prime}}^{v}\left\langle\boldsymbol{A}_{n q}^{*} \cdot\left(\boldsymbol{A}_{n^{\prime} q^{\prime}} \times \boldsymbol{A}_{n^{\prime \prime} q^{\prime \prime}}\right)\right\rangle-v \lambda_{n q}^{2} \xi_{n q}^{v},
$$

where $v$ is the fluid kinematic viscosity.

The method that we used is fully spectral. Apart from the mathematical esthetics of this modal decomposition, the numerical advantage of such a method is that no aliasing error is present, since multiplications are evaluated in spectral space using convolution products. A further advantage is that the precise modal structure is directly visible and the contributions of the different modes on the value of invariants such as angular momentum, helicity, and energy can be evaluated directly. Furthermore, the modes conserve exactly helicity and energy, even in the presence of a Galerkin truncation, thereby guaranteeing a perfect inviscid conservation of two important invariants of the system. An important disadvantage is, however, that for large numbers of modes the convolution products representing the nonlinearity of the Navier-Stokes equations become prohibitively expensive. For this reason, simulations of Reynolds numbers (based on the domain size) up to $10^{3}$ approximately will be reported in this work. Despite this limitation, it will be shown that the dynamics that can be considered using this technique allow us already to investigate a very rich turbulent system. The computational domain is shown in Fig. 1, with $R=L=2 \pi$. Time integration is performed using a fourth-order Runge-Kutta method. Further technical details on the method can be found in Refs. [46,50,54].

\section{B. Initial conditions}

To characterize the length scale of the flow-field, we define an effective wave number $\left|\lambda_{n q}\right|$ from expression (20), which allows us to determine an integral length scale by the relation

$$
l=\frac{\sum_{n, q}\left|\lambda_{n q}\right|^{-1} E(n, q)}{\sum_{n, q} E(n, q)},
$$

where $E(n, q)$ is the energy associated with mode $\xi_{n q}$. The integral time scale in our simulations is defined by $\tau=l / U$, where $l$ is given by expression (23). The Reynolds number is defined as $\operatorname{Re}=U L / v$, where $U=\sqrt{2 E / 3}$ and $L$ is the height of the domain. The simulations all start with an initial amount of kinetic energy $E=1.5707$, the viscosity is $v=0.01$, and the resolution is $n \times q=40 \times 60$ modes. In order to correctly resolve all scales 


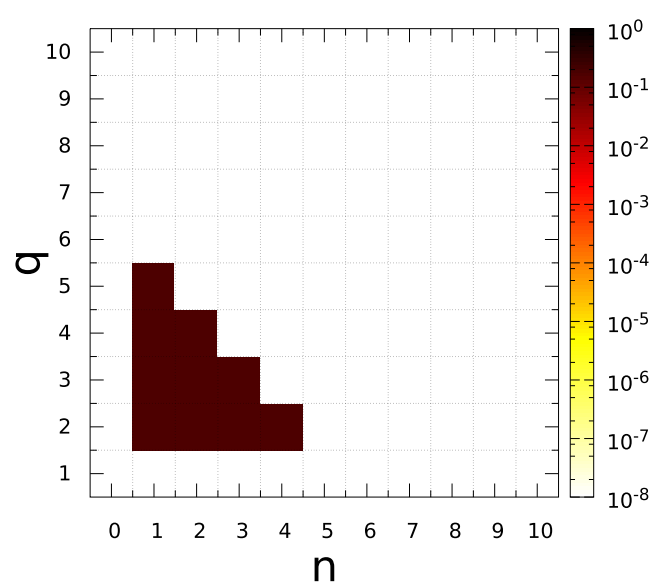

(a)

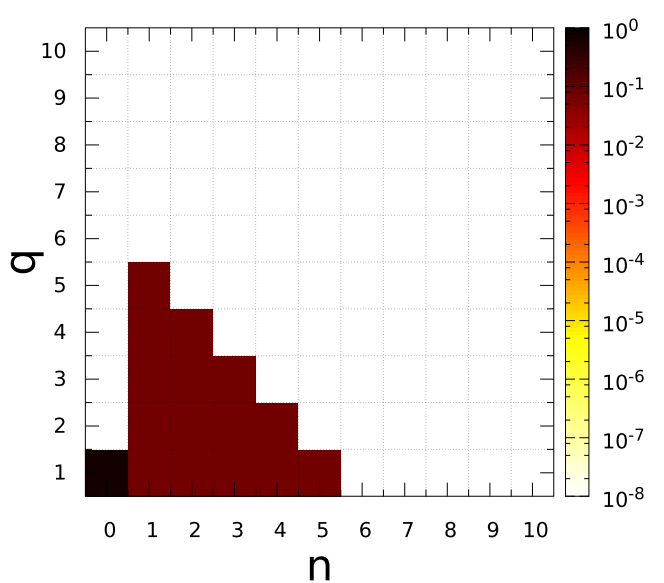

(b)

FIG. 2. The initial conditions corresponding to the runs documented in this paper. A zoom is shown on the range $n, q \leqslant 10$. (a) Case 1: zero initial $I_{1}$ and $H_{0}$. (b) Case 2: finite initial $I_{1}$ and $H_{0}$.

at all times, the Reynolds number characterizing the initial conditions is $\mathrm{Re}=643$ in the simulations.

Since this study reports on the first simulations of axisymmetric turbulence, the character of the investigation is necessarily exploratory and we do not claim to give an exhaustive description of all possible cases. We will rather concentrate on two sets of simulations, starting from random initial conditions with different initial values of the invariants. We have, among the infinite number of possible cases, chosen here to focus on cases where the initial energy distribution is centered around the large scales, i.e., low values of $n$ and $q$. Further studies with different initial conditions constitute a natural perspective of the present work.

Case 1. The angular momentum $I_{1}$ is zero at $t=0$. A comparable amount of energy is contained in the poloidal and toroidal directions, $E_{P} \approx E_{T}$ (the root-mean square values of $u_{r}, u_{\theta}$, and $u_{z}$ have the same order of magnitude). The resulting flow contains a finite amount of helicity $H_{1}$ and the circulation $H_{0}=0$.

Case 2. The angular momentum $I_{1}$ and circulation $H_{0}$ have finite values at $t=0$. Again a comparable amount of energy is contained in the poloidal and toroidal directions. The flow also contains a finite amount of helicity $H_{1}$. In particular, the mode $(n=0, q=1)$ is given an important amount of energy. The reason for this will be made clear in the following.

In Fig. 2, we show the energy distribution of the two initial conditions. It can be shown [54] by carefully considering the modal structure of the Chandrasekhar-Kendall decomposition that the total angular momentum $I_{1}$ and total circulation $H_{0}$ depend only on modes with $k_{n}=0$ (see, for instance, Ref. [46] for the circular analog of this). This difference is visible in the initial conditions in Fig. 2.

Table I reports the initial values of all the considered integral quantities, as well as their value, at $t=20 \tau$, in percentages $(\%)$ of their value at $t=0$.

\section{RESULTS}

In this section, we will discuss the results for two different cases. The first is the case where initially the angular momentum $I_{1}$ and the circulation $H_{0}$ are equal to zero and in which the initial toroidal and poloidal energies are comparable. In the second case, the initial energy is mainly concentrated in the largest scale $(n=0, q=1)$ with relatively strong initial azimuthal flow and a large initial value for the angular momentum. 
TABLE I. Initial values of the integral quantities and their values at $t=20 \tau$. The numbers in parentheses are the percentage (\%) of the values at $t=0$.

\begin{tabular}{lcccc}
\hline \hline & Case $1(t=0)$ & $(t=20 \tau)$ & Case 2 $(t=0)$ & $(t=20 \tau)$ \\
\hline$E$ & 1.5707 & $0.3884(24.7 \%)$ & 1.5707 & $0.6613(42.1 \%)$ \\
$H_{0}$ & 0.0 & $-0.0489(-)$ & 0.021 & $0.082(390.5 \%)$ \\
$H_{1}$ & 2.5535 & $0.2076(8.13 \%)$ & 0.676 & $0.314(46.4 \%)$ \\
$I_{1}$ & 0.0 & $0.0163(-)$ & 3.6 & $3.29(91.4 \%)$ \\
$I_{2}$ & 16.594 & $0.9468(5.71 \%)$ & 28.66 & $13.31(46.4 \%)$ \\
$E_{T}$ & 0.6805 & $0.0248(3.64 \%)$ & 1.0397 & $0.4655(44.77 \%)$ \\
$E_{P}$ & 0.8902 & $0.3636(40.84 \%)$ & 0.531 & $0.1958(36.87 \%)$ \\
$Z$ & 6.8446 & $0.1518(2.22 \%)$ & 2.361 & $0.2814(11.92 \%)$ \\
\hline \hline
\end{tabular}

\section{A. Case 1: A quasi-two-dimensional dynamics}

\section{Time evolution of the integral quantities}

An important question is how the different integral quantities will evolve as a function of time. Figure 3 shows the time evolution of some important integral quantities in case 1

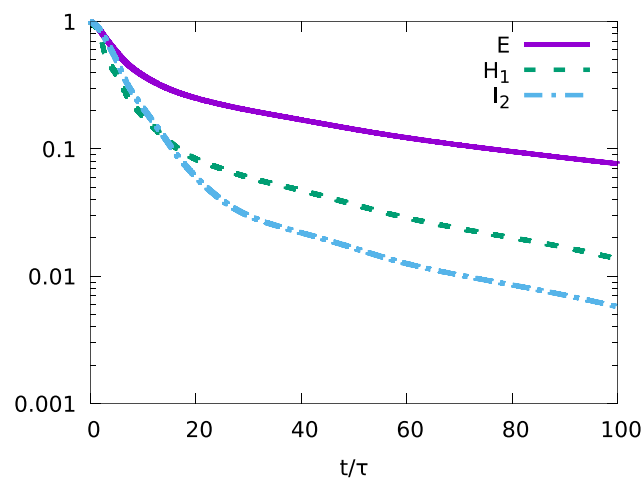

(a)

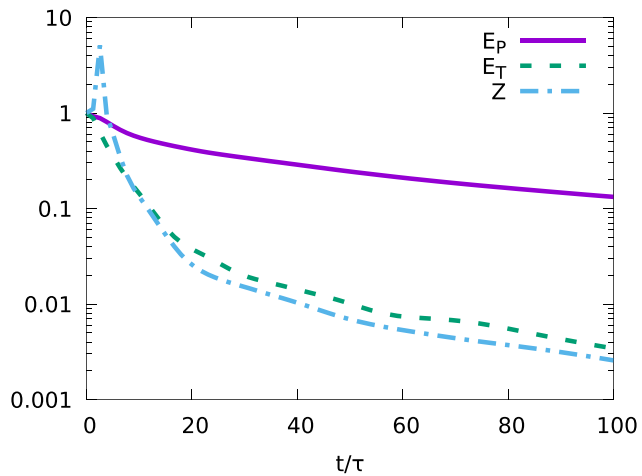

(c)

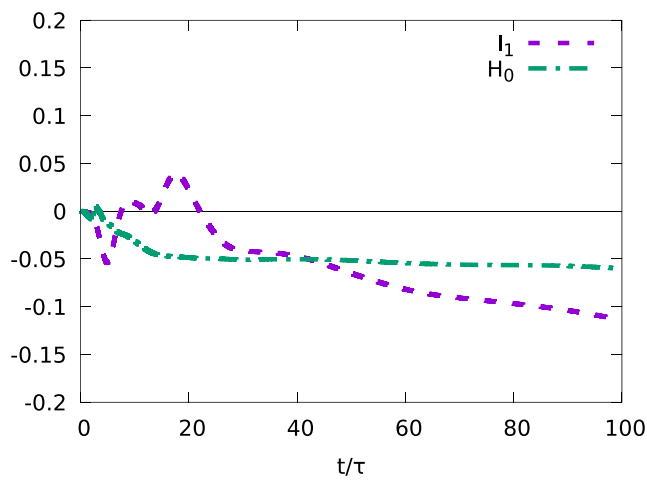

(b)

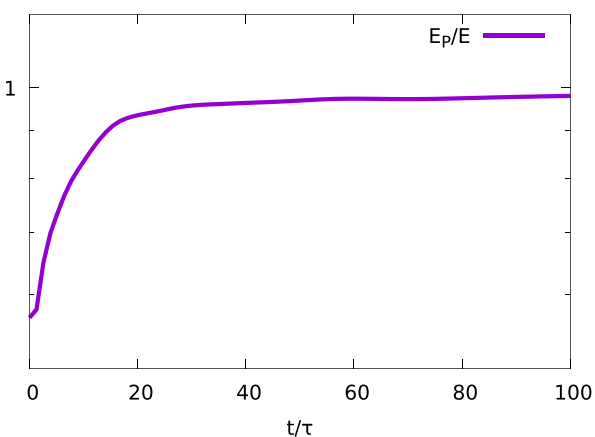

(d)

FIG. 3. Case 1. Time evolution of integral quantities, all normalized by their initial value except those for which the initial value is zero: (a) energy $E$, helicity $H_{1}$, and angular momentum fluctuations $I_{2}$; (b) circulation $H_{0}$ and angular momentum $I_{1}$; (c) toroidal and poloidal components of the energy, $E_{T}$ and $E_{P}$, and enstrophy $Z$; (d) ratio of poloidal to total energy. 


\section{DIRECT NUMERICAL SIMULATION OF AXISYMMETRIC TURBULENCE}

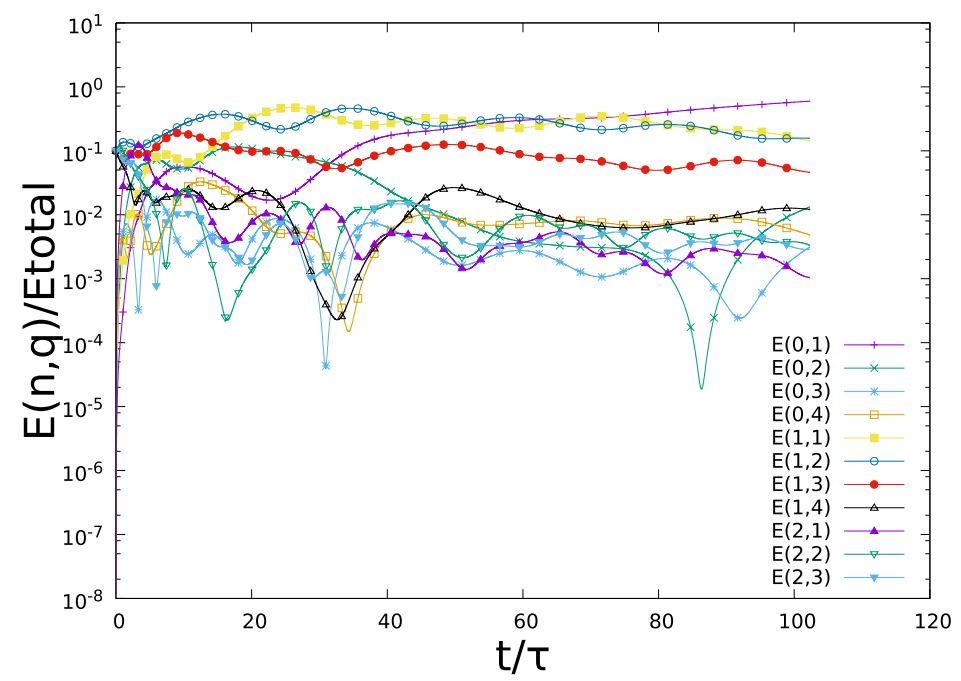

(a)

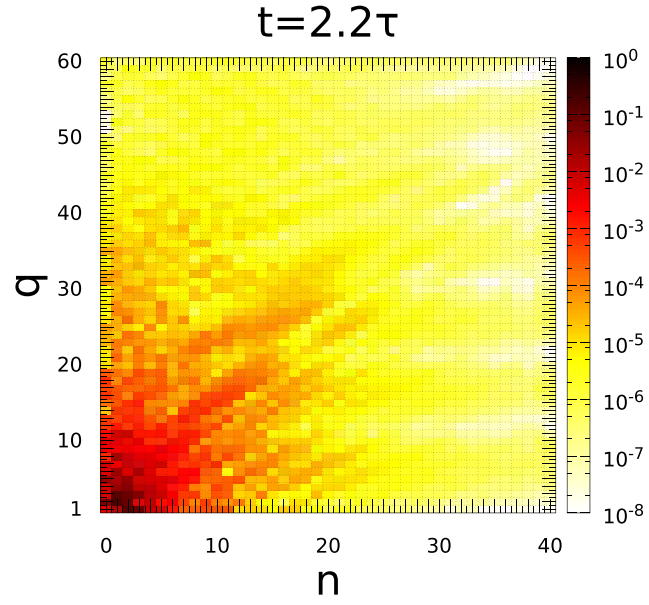

(b)

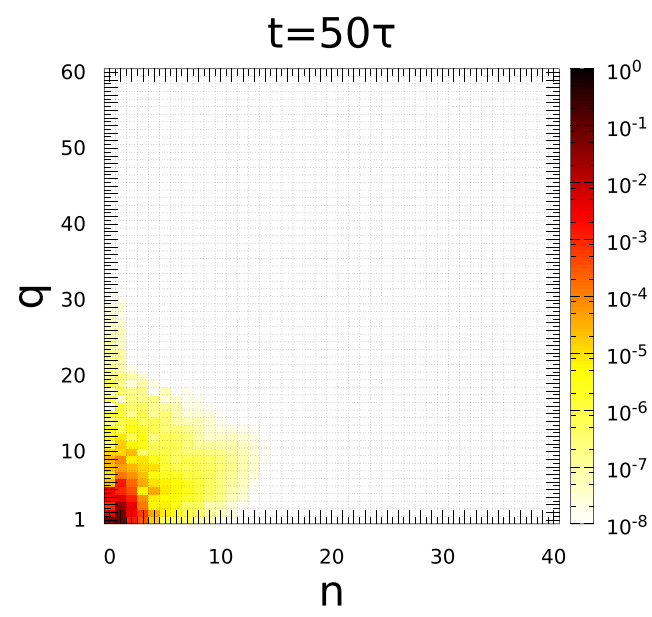

(c)

FIG. 4. Case 1. (a) Time evolution of modal energy for low-wave-number modes. Energy spectrum at (b) $t=2.2 \tau$ and (c) $t=50 \tau$.

$\left[H_{0}(t=0)=I_{1}(t=0)=0\right]$. Time evolutions are shown up to 100 initial turnover times $\tau$. It is clear from these results that the most robust quantity in these simulations is the poloidal kinetic energy. Helicity $H_{1}$, toroidal kinetic energy $E_{T}$, and the variance of angular momentum fluctuations $I_{2}$ all decay more rapidly. The angular momentum $I_{1}$ and circulation $H_{0}$ remain close to zero but are not exactly conserved. We now show that in the system considered these quantities can indeed be created by the combined effects of nonlinear coupling between the modes and viscous dissipation.

\section{Modal dynamics}

The generation of angular momentum can be understood by investigating the modal distribution of energy (Fig. 4). Only a small subset of modes, with small values of $n, q$ are shown. After a violent nonlinear phase for $t / \tau \lesssim 20$, where the energy is actively redistributed over a large number of modes, the modal activity relaxes and at long times, for $t / \tau \gtrsim 50$, the $(n=0, q=1)$ mode becomes dominant. This mode contains a finite value of angular momentum. In the inviscid case, its 

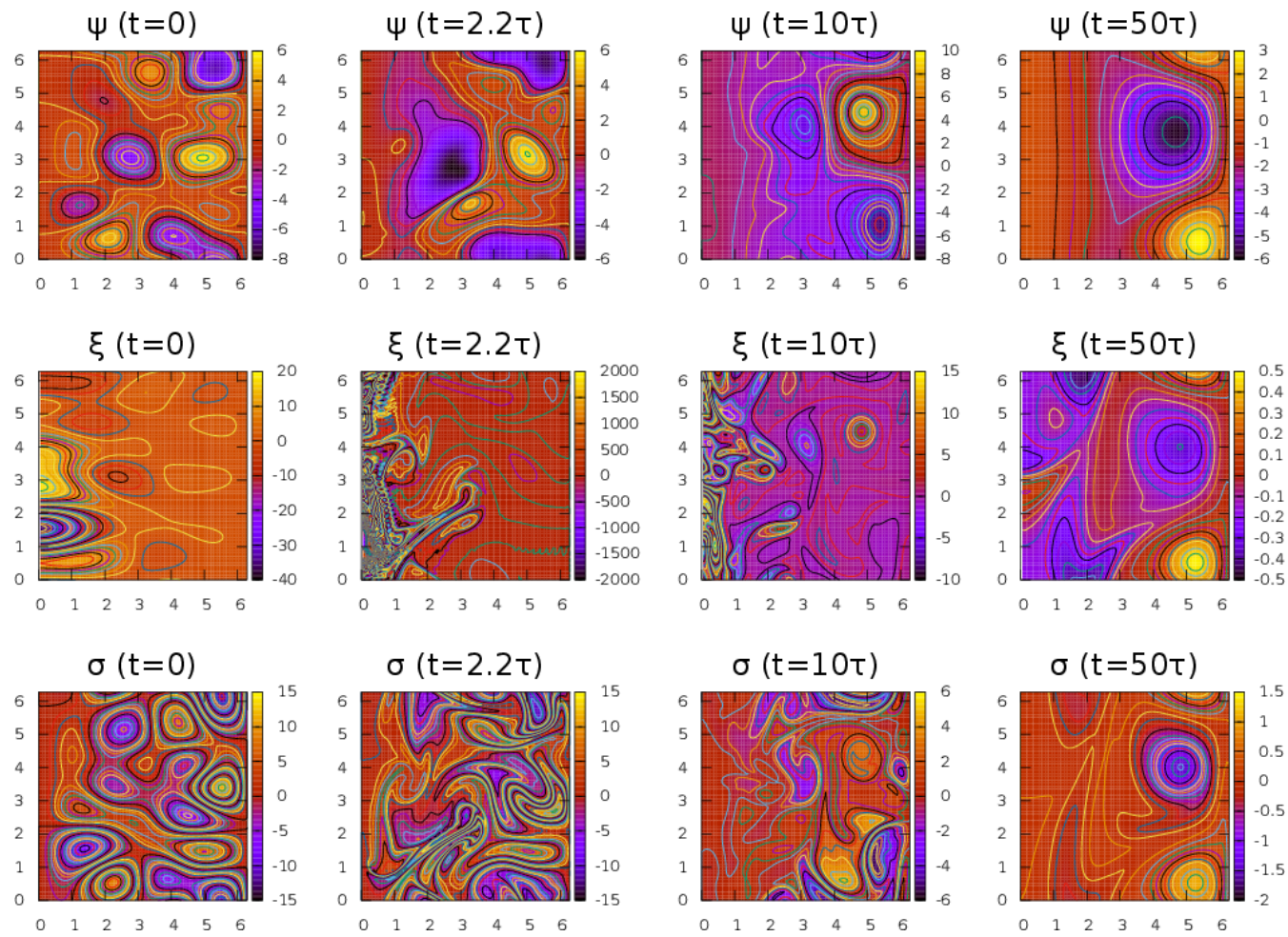

FIG. 5. Case 1. Flow visualizations. Isocontours of stream function, potential vorticity, and angular momentum in the $(r, z)$ plane at four different times: $t=0$ (initial field); $t=2.2 \tau$, where the turbulent interactions are strong; $t=10 \tau$ and $t=50 \tau$, where the large-scale coherent structures emerge.

angular momentum would be compensated by opposite contributions of modes with larger $q$ values. However, in the presence of viscosity, these modes are dissipated more rapidly than the low $q$ modes, which results in a net generation of angular momentum at large times. The same mechanism can also explain the generation of a finite amount of circulation $H_{0}$. It is worth noticing that the same mechanism was invoked for explaining the generation of angular momentum in $2 \mathrm{D}$ turbulence with circular rigid walls [44].

In Fig. 4, we also show the complete $n, q$ wave-number spectrum at two particular times. The first instant $t=2.2 \tau$ is in the phase of maximum modal activity: A large number of modes then contains the energy. At $t=50 \tau$ the system approaches its equilibrium state, and the energy is centered around a few low- $n, q$-number modes.

\section{Flow visualizations}

From Fig. 3 it can be seen that the flow becomes quasi-two-dimensional in the poloidal plane. It can then be expected that, if the kinetic energy in this plane is still sufficiently large, some metastable large structures should be observed in it, as in decaying 2D turbulence. Long-lived large-scale structures, dipolar-shaped, indeed form in the flow, as illustrated in Fig. 5, before viscosity dissipates the remaining kinetic energy. We show now that the corresponding flow is moreover a stationary solution of the Euler equations.

\section{Functional relations}

It was shown in Sec. II B that, in the quasi two-dimensional state obtained, the flow is a stationary solution of the axisymmetric Euler equations if and only if it obeys Eq. (15). To check if the 


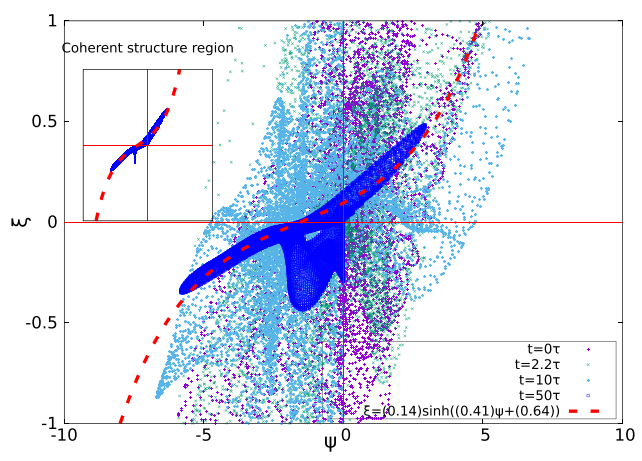

(a)

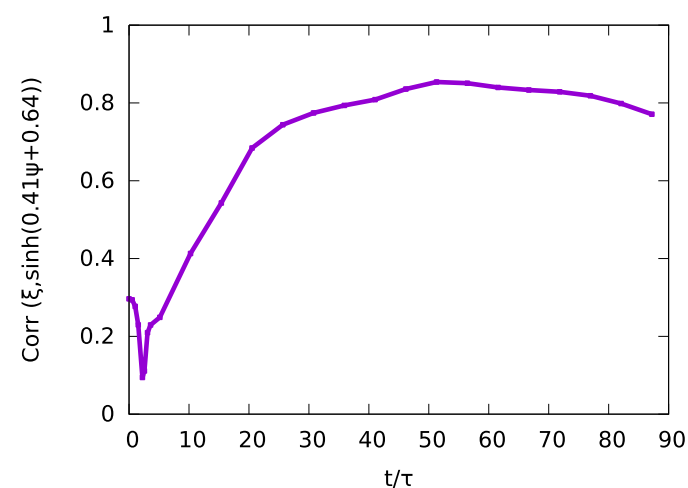

(b)

FIG. 6. Case 1. (a) Illustration by scatter plots of the time evolution of the correlation between $\xi$ and $\psi$. Inset: same scatter plot at $t=50 \tau$, plotted only in the coherent structure region $(r \geqslant 0.5 R)$. (b) Time evolution of the correlation coefficient (calculated on the whole domain) between $\xi$ and a hyperbolic sine function of $\psi$, $\sinh (0.41 \psi+0.64)$.

long-lived structures observed in Fig. 5 satisfy this relation, we plot in Fig. 6(a) the values of $\xi$ as a function of those of $\psi$ at different positions, and at different times. Remarkably, while the data display a significant scatter at the beginning of the simulation, a functional relation between $\xi$ and $\psi$ is clearly visible at long times $(t \approx 50 \tau)$, when the large structures are well defined in the flow.

At the lowest order, this relation is linear. Such a relation can be understood by using arguments based on a selective decay principle. Indeed, as shown in Fig. 3, the long-lived structures form after a much faster decay of the potential enstrophy $Z$ with respect to the energy. It is therefore likely that the corresponding state can be found variationally, by minimizing $Z$ with constrained $E$. Using the Lagrange multipliers method, this variational problem can be rewritten as

$$
\delta Z-\beta \delta E=0
$$

whose solutions satisfy a linear relation between $\xi$ and $\psi$ :

$$
\xi=\frac{\beta}{2} \psi
$$

These arguments explain the $\xi-\psi$ relation in Fig. 6 at the lowest order only. The relation observed is more precisely compatible with a hyperbolic sine function and is therefore similar to those predicted in 2D flows using statistical mechanics theories. The point-vortex approach indeed predicts in this sytem a relation $\omega \sim \sinh \psi$ [4-6]. The MRS theory predicts linear relations between the coarse-grained stream function and vorticity in the limit of strong mixing [12] or accounting for the conservation of the lowest order invariants only [13], but sinh or tanh relations between these quantities using first-order perturbative expansions [12] or accounting for the conservation of higher order invariants [14]. Given the similarity between Eq. (14) and the 2D Euler equations, it is therefore not surprising to obtain in the present system a sinh-like relationship between $\psi$ and $\xi$.

More quantitatively, it is shown in Fig. 6(b) that the correlation function between $\xi$ and $\sinh (0.41 \psi+0.64)$ increases up to $80 \%$ over the considered time interval. Finally, this functional relation between $\xi$ and $\psi$ is, as expected, even clearer if one considers only the values of these quantities in the coherent structure region, $r \geqslant 0.5 R$ (see right column of Fig. 5), as shown in the inset of Fig. 6(a).

\section{B. Case 2: A large-scale three-dimensional dynamics}

In two-dimensional turbulence, it was shown that the existence of an additional invariant can change the dynamics importantly. For instance, it was shown in a number of papers [44-46] that 


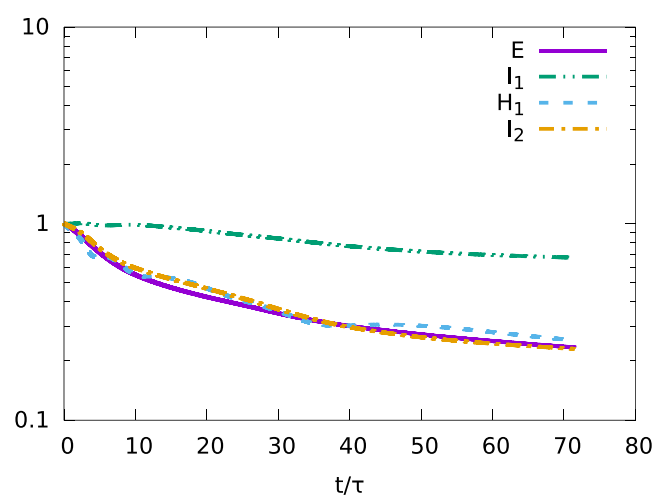

(a)

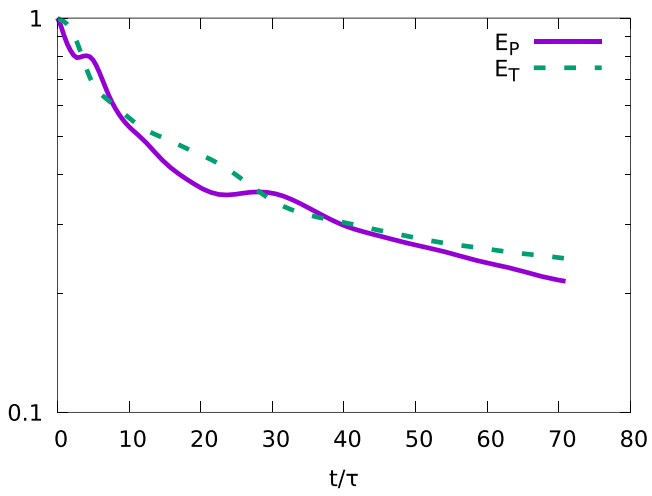

(b)

FIG. 7. Case 2. Time evolutions of the invariants (a) $E, I_{1}, H_{1}, I_{2}$ and (b) $E_{T}$ and $E_{P}$. All the quantities are normalized by their initial values.

the presence of a significant amount of angular momentum could change the final decaying state in a circular domain from a dipolar to a monopolar structure. Furthermore, in the framework of the statistical mechanics of the Euler equations, variational principles permit to show how the circulation can change the nature of the equilibrium solutions $[12,13]$ in a rectangular domain. In the present section, we therefore investigate this for axisymmetric turbulence, by considering initial conditions containing a substantial amount of angular momentum $I_{1}$.

To generate an initial condition with substantial angular momentum, the $(n=0, q=1)$ mode was initialized with an amount of energy large compared to that of the other modes [see Fig. 2(b)]. The reason for this is that we have seen in case 1 that this mode becomes energetic even if it is not so initially. In case 1, this effect was only apparent at the end of the simulation, where the Reynolds number was already relatively low. In order to investigate its influence in the nonlinearly most active phase of the time evolution, we give it a large value initially.

We stress here that the way to impose the angular momentum is not unique. All $(n=0)$ modes contribute to the angular momentum. We have chosen to add the angular momentum by acting on the largest scale $(n=0, q=1)$, which is the least dissipative mode of the system. It is thus not only the angular momentum which is forced by this choice, but also the fact that the largest possible structure of the flow contains a fair amount of energy. We have also carried out tests in which the angular momentum was injected at higher wave numbers, and the three-dimensional structure was in that case less robust. However, in large-scale forced real-life flow, such as the Von Kármán flow, it is also by acting on the large-scale modes that angular momentum can be injected, which partly justifies our choice.

\section{Time evolution of the integral quantities}

We see in Fig. 7 that the most robust quantity is the angular momentum $I_{1}$. The toroidal and poloidal components of the energy decay at nearly the same pace. This shows that we are now in a case different from the former quasi-2D scenario, where the poloidal component of energy was much better conserved than the toroidal one. The evolution of $H_{0}$ is not shown here. Its initial value is small and, as in case 1, it increases in time to a value still negligible compared to the other invariants.

We further see that the helicity $H_{1}$ is better conserved than in the previous case. Note that $H_{1}$ is zero in $2 \mathrm{D}$ turbulence. Therefore, the fact that this three-dimensional quantity does not rapidly vanish indicates that we have a three-dimensional flow. Moreover, by definition $H_{1}=\left\langle u_{\theta} \omega_{\theta}\right\rangle$ and therefore $\left|H_{1}\right| \leqslant\left\langle u_{\theta}^{2}\right\rangle^{1 / 2}\left\langle\omega_{\theta}^{2}\right\rangle^{1 / 2}$. Since in the present run $E_{T} \equiv\left\langle u_{\theta}^{2}\right\rangle / 2$ does not decay as rapidly as in case $1, H_{1}$ is not constrained to decay that rapidly. 


\section{DIRECT NUMERICAL SIMULATION OF AXISYMMETRIC TURBULENCE}

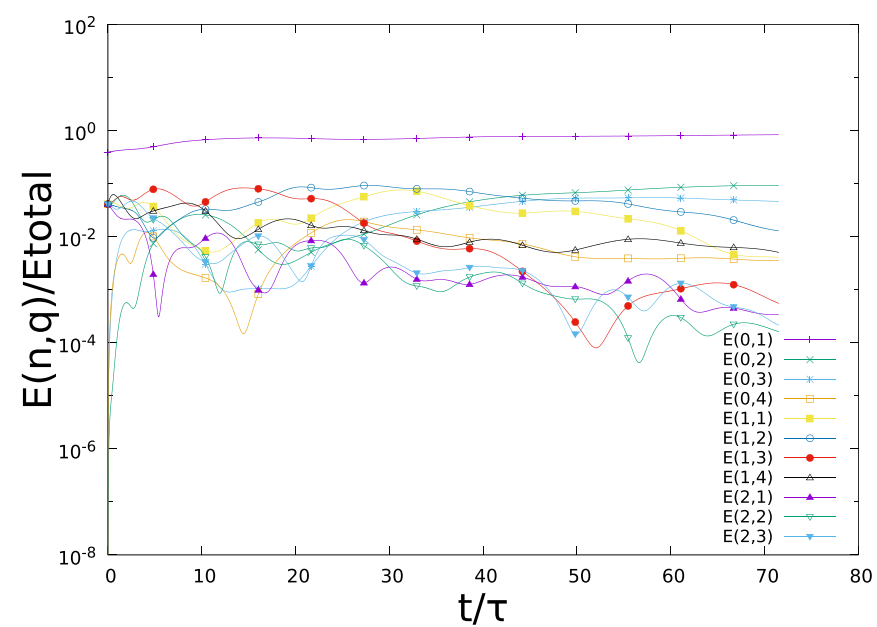

FIG. 8. Case 2. Time evolution of the modal energy normalized by the total energy for low-wave-number modes.

\section{Modal dynamics}

The modal distribution of energy in this scenario is shown in Fig. 8. We see that the largest scale mode $(n=0, q=1)$ contains during the whole simulation a significant portion of the total energy. This portion incidentally increases in time. The $(n=0, q=1)$ mode contains both toroidal and poloidal energy, and the fact that it dominates the total energy explains why the ratio of toroidal to poloidal energy remains approximately constant. The energy of the other modes decays more rapidly than that of the $(n=0, q=1)$ mode, but this does not strongly influence the large-scale dynamics.

\section{Flow visualizations}

Figure 9 shows that a large-scale, long-lived structure also forms in this case. Its shape is monopolar. At the latest time considered, some tendency is observed toward an axially invariant flow, compatible with the dominant $(n=0, q=1)$ mode. It would be interesting to consider boundary conditions breaking the periodicity in the axial direction (e.g., closed cylindrical domain), since then other modes than the $n=0$ ones might become energetically dominant as well at long times. This certainly motivates further studies.

\section{Functional relations}

To further characterize this well-organized flow, we now look for relations of the types (16) and (17) between the variables $\sigma, \xi$, and $\psi$. Such relations were shown to characterize the stationary
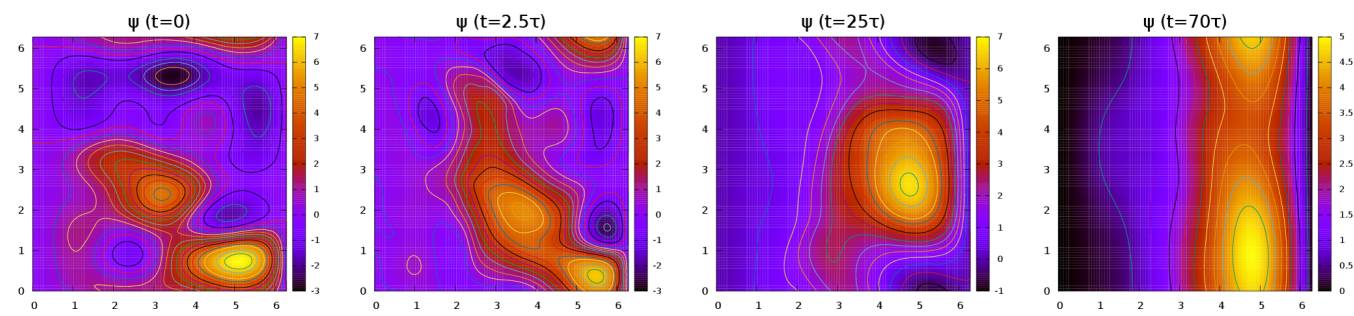

FIG. 9. Case 2. Flow visualizations. Isocontours of the stream function in the $(r, z)$ plane at four different times. 


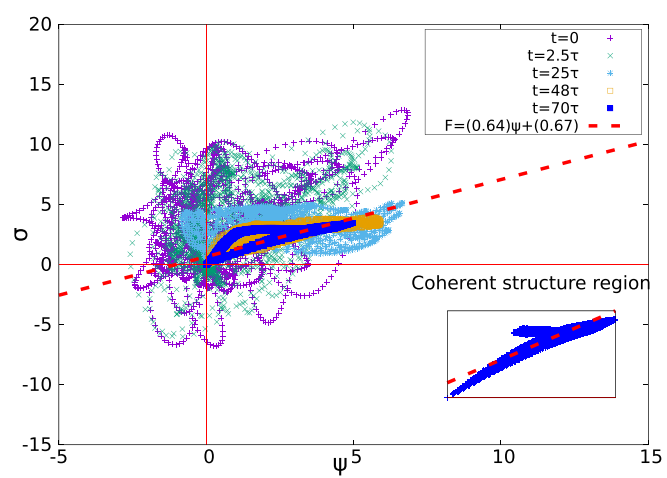

(a)

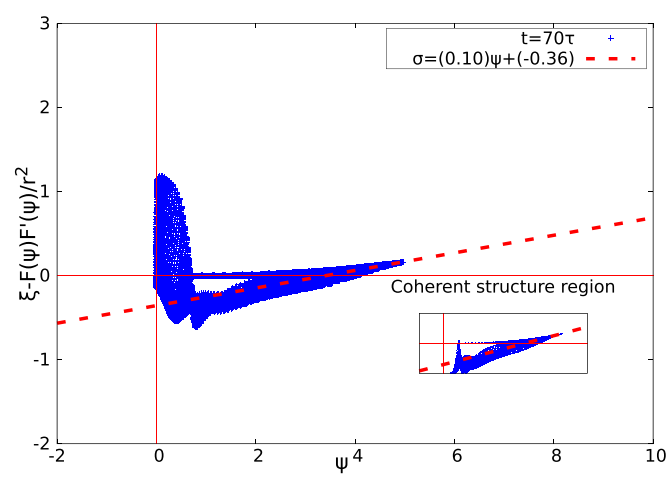

(b)

FIG. 10. Case 2. (a) Scatter plots of $\psi$ and $\sigma$ at different times. (b) Scatter plot of $\psi$ and $\xi-F(\psi) F^{\prime}(\psi) / r^{2}$, where $F$ is the affine function fitted from the data $\psi$ vs $\sigma$ at $t=70 \tau$. The dashed lines are the functions calculated by fitting the data by affine functions at $t=70 \tau$. Insets: scatter plots at $t=70 \tau$ in the coherent structure region, $r \geqslant 0.5 R$.

solutions of the axisymmetric Euler equations in the nondegenerate, swirling case. In Fig. 10 we see some evidence of the existence of functions $F(\psi)=\sigma$ and $G(\psi)=\xi-F^{\prime} F / r^{2}$ at $t=70 \tau$. However, at the same time the functional relation $G$ between $\psi$ and $\xi-F F^{\prime}(\psi) / r^{2}$ is not as clear as that between $\psi$ and $\sigma(F)$. If one only focuses on the field with $r>0.5 R$, where the monopole is present (see right panel of Fig. 9), one observes a clearer relation between these quantities. This means that the coherent structures of this viscous flow are solutions of the Euler equations. Moreover, the resulting functions $F$ and $G$ are close to linear, which means that the quasisteady flow obtained is close to a Beltrami state [38].

We now interpret this result by using arguments based on a selective decay principle, as we did for case 1. According to Fig. 7, in case 2 the long-lived structures form after a decay of $E, H_{1}$, and $I_{2}$ much faster than that of the angular momentum. The corresponding flow can therefore be presumably predicted variationally, by minimizing a quantity $E+\alpha H_{1}+\beta I_{2}$ with constrained $I_{1}$. Using the Lagrange multipliers method, such a problem can be rewritten as

$$
\delta\left(E+\alpha H_{1}+\beta I_{2}\right)-\gamma \delta I_{1}=0 .
$$

The solutions of this equation satisfy the relations:

$$
\begin{gathered}
\sigma=-\frac{1}{\alpha} \psi, \\
\xi+\frac{1}{\alpha} \frac{\sigma}{r^{2}}=\frac{2 \beta}{\alpha^{2}} \psi+\frac{\gamma}{\alpha},
\end{gathered}
$$

which can be rewritten in the form of Eqs. (16) and (17) after setting $F(\psi)=-(1 / \alpha) \psi$ and $G(\psi)=\left(2 \beta / \alpha^{2}\right) \psi+(\gamma / \alpha)$. These expressions of the functions $F$ and $G$ are compatible with those exhibited by the flow at $t=70 \tau$ (see Fig. 10).

\section{DISCUSSION}

\section{A. Case 1: Two-and-a-half-dimensional turbulence}

The first case we considered in this investigation, with small initial angular momentum, finds its analogy in the case of quasistatic magnetohydrodynamics (QS-MHD). In this dynamics, characteristic of liquid metals in the presence of a strong imposed magnetic field, the influence of the magnetic field on the flow is well approximated by an anisotropic damping term. After a linear phase, in which anisotropy develops [55], the dynamics are well represented by two-and-a-half-dimensional turbulence [56], that is, a flow invariant along the direction of the magnetic field $\mathbf{B}$, and in which $u_{\|}$, 
the component of the velocity field aligned with $\mathbf{B}$ is advected as a passive scalar by the velocity field perpendicular to the magnetic field, $\boldsymbol{u}_{\perp}$. In this case, the dynamics of the two fields, $u_{\|}$and $\boldsymbol{u}_{\perp}$ become independent, and follow the equations

$$
\begin{aligned}
\partial_{t} u_{\|}+\left\{\psi_{\perp}, u_{\|}\right\} & =0, \\
\partial_{t} \omega_{\perp}+\left\{\psi_{\perp}, \omega_{\perp}\right\} & =0,
\end{aligned}
$$

where $\Delta \psi_{\perp}=-\omega_{\perp}$ and $\omega_{\perp}$ and $\psi_{\perp}$ are the vorticity and stream function associated with the poloidal velocity $\boldsymbol{u}_{\perp}$. This system is equivalent to the present case of axisymmetric turbulence, with the correspondence $(\sigma, \xi, \psi) \leftrightarrow\left(u_{\|}, u_{\perp}, \psi_{\perp}\right)$, if the RHS term of Eq. (5) is neglected. In the QS-MHD case, the dynamics of the parallel flow can then be well described by a direct cascade process, where the variance of $u_{\|}$rapidly decays [57] and the perpendicular dynamics is characterized by a long-lasting two-dimensional turbulent state (if the viscosity is sufficiently small). The inviscid dynamics are then described by equations similar to Eq. (4) and (5), with zero right-hand sides. It is therefore not surprising that in the present case we observe at long times a two-dimensional dynamics in the poloidal plane if the initial value of the angular momentum fluctuations $\sigma$ is weak, that is, if the right-hand side of Eq. (5) can be neglected.

To consolidate this picture, we have carried out two supplementary tests.

First, to check if it is not simply a viscous effect which leads to the dominance of the poloidal energy, we also carried out a simulation with the same initial conditions as case 1, but starting off with a smaller Reynolds number, $\operatorname{Re} \approx 6$. In this case, both the poloidal and toroidal energy drop simultaneously over time, showing that purely viscous interactions cannot explain the observed behavior (for more details on this simulation, we refer to Ref. [54]).

Second, to verify whether the poloidal energy will increase even if it is initially small, we have carried out a simulation with initially more than $97 \%$ of the energy contained in the toroidal component. At the modest Reynolds number considered, the poloidal energy increases, proportionally, from $2 \%$ to $16 \%$ over the time interval $0<t<100 \tau$. This seems to indicate that the axisymmetric system, in the absence of forcing terms, tends to a quasi-two-dimensional dynamics in the poloidal plane, even though higher Reynolds numbers are desirable to certify this conjecture (for more details, we refer to Ref. [54]).

\section{B. Case 2: Unimodal dynamics}

The second case, in which the $(n=0, q=1)$ mode was initially strong, showed that this same mode remained dominant during the whole dynamics. The difference with the first case is striking, since this mode is three dimensional. No quasi-2D flow state was attained. The flow visualizations showed that the long-time pattern of the flow contained a monopolar structure, different from the dipolar structure in case 1.

The situation is quite similar to the work of $\mathrm{Li}$ and Mongomery [44,46], where it was shown in two-dimensional turbulence in a circular domain (where the angular momentum is also an invariant of the inviscid system) that the final, long-lasting state could drastically change depending on whether the initial conditions contained, or not, a significant amount of angular momentum.

It is interesting that the resulting structure at $t=25 \tau$ is monopolar rather than axially invariant, a state which could have been obtained since the dominant mode is axially invariant $(n=0)$. The other modes have therefore a nontrivial contribution to the flow. At later times $(t=70 \tau)$, the axial invariance of the $(n=0, q=1)$ mode seems to become visible in the flow visualizations, a feature definitely highly sensitive to the boundary conditions on the top and the bottom of the domain.

Some agreement with theoretical predictions was observed in the scatter plots of the different quantities, but this needs simulations at higher Reynolds numbers for confirmation. 


\section{CONCLUSION}

We have carried out a numerical investigation of the properties of freely decaying axisymmetric turbulence. Two sets of initial conditions have been considered, showing several key features of the system. Obviously this study, which is the first numerical investigation of axisymmetric turbulence, cannot consider all different possible situations, and we have therefore focused on the specific case of initial conditions with energy contained in the large-scale modes only. What we have learned from these simulations is that axisymmetric turbulence seems to form large coherent structures, consistent with an inverse cascade mechanism. Mean angular momentum is a very robust invariant, but angular momentum fluctuations $I_{2}$ and the associated toroidal velocity fluctuations $E_{T}$ decay more rapidly than poloidal ones, in agreement with a direct cascade scenario for the toroidal energy.

We can now compare our results to some predictions of the theories based on statistical mechanics at equilibrium. First, we have found numerically that helicity is a weak invariant of the axisymmetric Euler equations, as was proposed in Ref. [33] and unlike the assumptions used in Refs. [30,31]. We note here that we have performed with the same numerical method a detailed investigation (reported in a separate manuscript) of the turbulent cascades arising in axisymmetric flows, which supports this result as well. Second, the dipolar and monopolar structures arising in our simulations show a clear resemblance with the predictions in Ref. [32]. However, the axial boundary conditions in the two setups being distinct (periodic in our case, closed in Ref. [32]), no closer comparison between the numerical and theoretical results can be made. This prevents also any comparison of our results with those of Ref. [33], which were derived in a Taylor-Couette geometry. The numerical investigation of the dynamics of the axisymmetric Navier-Stokes equations in a cylinder with confining solid boundaries at the top and the bottom is ongoing, and we hope that it will clarify which theoretical framework most adequately describes axisymmetric turbulence.

It is also tempting to compare our results with those obtained experimentally for the von Kármán flow, although we have already stressed the fact that the latter is only statistically axisymmetric. Any comparison of it with strictly axisymmetric turbulence would be therefore possible at a phenomenological level only. However, even at this level both systems are very different. First, the von Kármán flow is not periodic in the axial direction, since the impellers continuously break the $z$ invariance. Furthermore, in the experiment a certain mean helicity is somehow imposed: This constraint does not stem from the equations and their invariants but is rather imposed by the forcing geometry. Finally, in the experiments the toroidal velocity fluctuations were observed to be strong, whereas in the freely decaying simulations they did not seem to be very robust. Again, the forcing in the experiment might explain these observations, since the impellers introduce a dominantly toroidal energy. We plan to perform a numerical investigation similar to that presented in this paper but with a forcing similar to the von Kármán setup.

More generally, this exploratory work opens a vast number of perspectives and questions. For instance, how can the tendency to bidimensionalization be reconciled with the dominance of a three-dimensional $(n=0, q=1)$ mode at long times? How do the boundary conditions influence the results? What is the effect of forcing on the observations? Can we determine the cascade directions of the different invariants? How do the results evolve at larger Reynolds numbers?

\section{ACKNOWLEDGMENTS}

Shuojun Li is gratefully acknowledged for providing his code for two-dimensional turbulence. Bérengère Dubrulle is acknowledged for insightful comments and for suggesting that we carry out this investigation. We thank the referees for their very valuable comments, which helped us to improve the paper. B.Q. was supported by a grant from the China Scholarship Council. All simulations were carried out using the facilities of the PMCS2I (École Centrale de Lyon). 


\section{DIRECT NUMERICAL SIMULATION OF AXISYMMETRIC TURBULENCE}

[1] F. T. M. Nieuwstadt and J. A. Steketee, Selected Papers of J. M. Burgers (Kluwer, Dordrecht, 1995).

[2] L. Onsager, Statistical hydrodynamics, Nuovo Cimento 6, 279 (1949).

[3] G. L. Eyink and K. R. Sreenivasan, Onsager and the theory of hydrodynamic turbulence, Rev. Mod. Phys. 78, 87 (2006).

[4] D. Montgomery, Two-dimensional vortex motion and "negative temperatures," Phys. Lett. A 39, 7 (1972).

[5] G. Joyce and D. Montgomery, Negative temperature states for the two-dimensional guiding center plasma, J. Plasma Phys. 10, 107 (1973).

[6] D. C. Montgomery and G. Joyce, Statistical mechanics of "negative temperature" states, Phys. Fluids 17, 1139 (1974).

[7] T. S. Lundgren and Y. B. Pointin, Statistical mechanics of two-dimensional vortices, J. Stat. Phys. 17, 323 (1977).

[8] R. H. Kraichnan and D. Montgomery, Two-dimensional turbulence, Rep. Prog. Phys. 43, 547 (1980).

[9] D. Montgomery, W. H. Matthaeus, W. T. Stribling, D. Martinez, and S. Oughton, Relaxation in two dimensions and the "sinh-Poisson" equation, Phys. Fluids A 4, 3 (1992).

[10] J. Miller, Statistical Mechanics of Euler Equations in Two Dimensions, Phys. Rev. Lett. 65, 2137 (1990).

[11] R. Robert and J. Sommeria, Statistical equilibrium states for two-dimensional flows, J. Fluid Mech. 229, 291 (1991).

[12] P. H. Chavanis and J. Sommeria, Classification of self-organized vortices in two-dimensional turbulence: The case of a bounded domain, J. Fluid Mech. 314, 267 (1996).

[13] A. Naso, P.-H. Chavanis, and B. Dubrulle, Statistical mechanics of two-dimensional Euler flows and minimum enstrophy states, Eur. Phys. J. B 77, 187 (2010).

[14] W. Qi and J. B. Marston, Hyperviscosity and statistical equilibria of Euler turbulence on the torus and the sphere, J. Stat. Mech. (2014) P07020.

[15] E. Segre and S. Kida, Late states of incompressible 2D decaying vorticity fields, Fluid Dyn. Res. 23, 89 (1998).

[16] A. Venaille, T. Dauxois, and S. Ruffo, Violent relaxation in two-dimensional flows with varying interaction range, Phys. Rev. E 92, 011001 (2015).

[17] T. Warn, Statistical mechanical equilibria of the shallow water equations, Tellus A 38, 1 (1986).

[18] P.-H. Chavanis and J. Sommeria, Statistical mechanics of the shallow water system, Phys. Rev. E 65, 026302 (2002).

[19] A. Renaud, A. Venaille, and F. Bouchet, Equilibrium statistical mechanics and energy partition for the shallow water model, J. Stat. Phys. 163, 784 (2016).

[20] P. B. Weichman, Competing turbulent cascades and eddy-wave interactions in shallow water equilibria, Phys. Rev. Fluids 2, 034701 (2017).

[21] F. Bouchet and J. Sommeria, Emergence of intense jets and Jupiter's Great Red Spot as maximum-entropy structures, J. Fluid Mech. 464, 165 (2002).

[22] P.-H. Chavanis, Statistical mechanics of geophysical turbulence: Application to jovian flows and Jupiter's great red Spot, Phys. D (Amsterdam, Neth.) 200, 257 (2005).

[23] F. Bouchet and A. Venaille, Statistical mechanics of two-dimensional and geophysical flows, Phys. Rep. 515, 227 (2012).

[24] A. Venaille and F. Bouchet, Statistical Ensemble Inequivalence and Bicritical Points for Two-Dimensional Flows and Geophysical Flows, Phys. Rev. Lett. 102, 104501 (2009).

[25] A. Naso, P.-H. Chavanis, and B. Dubrulle, Statistical mechanics of Fofonoff flows in an oceanic basin, Eur. Phys. J. B 80, 493 (2011).

[26] K. R. Sreenivasan, On the scaling of the turbulence energy dissipation rate, Phys. Fluids 27, 1048 (1984).

[27] Y. Kaneda, T. Ishihara, M. Yokokawa, K. Itakura, and A. Uno, Energy dissipation rate and energy spectrum in high resolution direct numerical simulations of turbulence in a periodic box, Phys. Fluids 15, L21 (2003).

[28] K. Mohseni, Statistical equilibrium theory for axisymmetric flows: Kelvin's variational principle and an explanation for the vortex ring pinch-off process, Phys. Fluids 13, 1924 (2001).

[29] C. C. Lim, Coherent structures in an energy-enstrophy theory for axisymmetric flows, Phys. Fluids 15, $478(2003)$ 
[30] N. Leprovost, B. Dubrulle, and P.-H. Chavanis, Dynamics and thermodynamics of axisymmetric flows: Theory, Phys. Rev. E 73, 046308 (2006).

[31] A. Naso, R. Monchaux, P. H. Chavanis, and B. Dubrulle, Statistical mechanics of Beltrami flows in axisymmetric geometry: Theory reexamined, Phys. Rev. E 81, 066318 (2010).

[32] A. Naso, S. Thalabard, G. Collette, P.-H. Chavanis, and B. Dubrulle, Statistical mechanics of Beltrami flows in axisymmetric geometry: equilibria and bifurcations, J. Stat. Mech. (2010) P06019.

[33] S. Thalabard, B. Dubrulle, and F. Bouchet, Statistical mechanics of the 3d axisymmetric Euler equations in a Taylor-Couette geometry, J. Stat. Mech. (2014) P01005.

[34] A. Pumir and E. D. Siggia, Finite-Time Singularities in the Axisymmetric Three-Dimension Euler Equations, Phys. Rev. Lett. 68, 1511 (1992).

[35] R. Grauer and T. C. Sideris, Finite time singularities in ideal fluids with swirl, Phys. D (Amsterdam, Neth.) 88, 116 (1995).

[36] P. J. Zandbergen and D. Dijkstra, Von Kármán swirling flows, Annu. Rev. Fluid Mech. 19, 465 (1987).

[37] F. Ravelet, L. Marié, A. Chiffaudel, and F. Daviaud, Multistability and Memory Effect in a Highly Turbulent Flow: Experimental Evidence for a Global Bifurcation, Phys. Rev. Lett. 93, 164501 (2004).

[38] R. Monchaux, F. Ravelet, B. Dubrulle, A. Chiffaudel, and F. Daviaud, Properties of Steady States in Turbulent Axisymmetric Flows, Phys. Rev. Lett. 96, 124502 (2006).

[39] H. K. Moffatt and A. Tsinober, Helicity in laminar and turbulent flow, Annu. Rev. Fluid Mech. 24, 281 (1992).

[40] S. Thalabard, B. Saint-Michel, E. Herbert, F. Daviaud, and B. Dubrulle, A statistical mechanics framework for the large-scale structure of turbulent von Kármán flows, New J. Phys. 17, 063006 (2015).

[41] I. Delbende, M. Rossi and B. Piton, Direct numerical simulation of helical vortices, Int. J. Eng. Systems Model. Simulation 4, 94 (2012).

[42] $H_{1}=\langle\sigma \xi\rangle=\left\langle u_{\theta} \omega_{\theta}\right\rangle$ is, strictly speaking, half the total helicity $\langle\mathbf{u} \cdot \boldsymbol{\omega}\rangle$, since $\left\langle u_{\theta} \omega_{\theta}\right\rangle=\left\langle u_{r} \omega_{r}+u_{z} \omega_{z}\right\rangle$.

[43] A. Szeri and P. Holmes, Nonlinear stability of axisymmetric swirling flows, Philos. Trans. R. Soc. London, Ser. A 326, 327 (1988).

[44] S. Li, D. Montgomery, and W. B. Jones, Inverse cascades of angular momentum, J. Plasma Phys. 56, 615 (1996).

[45] S. Li and D. Montgomery, Decaying two-dimensional turbulence with rigid walls, Phys. Lett. A 218, 281 (1996).

[46] S. Li, D. C. Montgomery, and W. P. Jones, Two-dimensional turbulence with rigid walls, Theor. Comput. Fluid Dyn. 9, 167 (1997).

[47] S. Chandrasekhar and P. C. Kendall, On force-free magnetic fields, Astrophys. J. 126, 457 (1957).

[48] D. Montgomery, L. Turner, and G. Vahala, Three-dimensional magnetohydrodynamic turbulence in cylindrical geometry, Phys. Fluids (1958-1988) 21, 757 (1978).

[49] H. Chen, X. Shan, and D. Montgomery, Galerkin approximations for dissipative magnetohydrodynamics, Phys. Rev. A 42, 6158 (1990).

[50] X. Shan, D. C. Montgomery, and H. Chen, Nonlinear magnetohydrodynamics by Galerkin-method computation, Phys. Rev. A 44, 6800 (1991).

[51] X. Shan and D. C. Montgomery, Magnetohydrodynamic Stabilization Through Rotation, Phys. Rev. Lett. 73, 1624 (1994).

[52] P. D. Mininni, D. C. Montgomery, and L. Turner, Hydrodynamic and magnetohydrodynamic computations inside a rotating sphere, New J. Phys. 9, 303 (2007).

[53] J. Boisson and B. Dubrulle, Three-dimensional magnetic field reconstruction in the VKS experiment through Galerkin transforms, New J. Phys. 13, 023037 (2011).

[54] B. Qu, Numerical simulation of axisymmetric turbulence, Ph.D. thesis, Université de Lyon, France, 2017.

[55] H. K. Moffatt, On the suppression of turbulence by a uniform magnetic field, J. Fluid Mech. 28, 571 (1967).

[56] D. Montgomery and L. Turner, Two-and-a-half-dimensional magnetohydrodynamic turbulence, Phys. Fluids 25, 345 (1982).

[57] B. Favier, F. S. Godeferd, C. Cambon, A. Delache, and W. J. T. Bos, Quasi-static magnetohydrodynamic turbulence at high Reynolds number, J. Fluid Mech. 681, 434 (2011). 